\title{
SELEÇÃO DE ESPÉCIES DA FLORESTA OMBRÓFILA DENSA E INDICAÇÃO DA DENSIDADE DE PLANTIO NA RESTAURAÇÃO FLORESTAL DE ÁREAS DEGRADADAS NA AMAZÔNIA
}

\author{
SELECTION OF SPECIES FROM NATURAL FOREST AND INDICATION OF PLANTING \\ DENSITY FOR RECLAMATION OF DEGRADED AREAS IN THE AMAZON FOREST
}

\author{
Rafael Paiva Salomão ${ }^{1}$ Antônio Cordeiro Santana ${ }^{2}$ Sílvio Brienza Júnior ${ }^{3}$
}

RESUMO

A identificação de espécies-chave que influenciam a ocorrência das demais espécies associadas é de fundamental importância para o sucesso da restauração florestal em áreas degradadas. Objetivou-se neste trabalho apresentar um índice fitossociológico e socioeconômico (IFSE), obtido por técnicas de análise fatorial, cujo modelo envolveu seis variáveis quantitativas (abundância, frequência, dominância, biomassa, valor comercial da madeira e quantidade de produtos florestais não madeireiros) e igual número de variáveis qualitativas, cuja variável dummy assumiu valor igual a 1 para aquelas espécies cujos maiores valores acumulados equivaleram a $50 \%$ do total para cada uma das variáveis quantitativas analisadas. A amostra para o inventário florestal em 407 ha de floresta ombrófila densa foi composta por 80 parcelas de 0,25 ha $(4,91 \%$ de intensidade amostral) onde todos os indivíduos com diâmetro a 1,30 m do solo (DAP) $\geq 10$ $\mathrm{cm}$ foram identificados e mensurados. Foram registrados 10.105 indivíduos, distribuídos em 493 espécies, abrangendo 58 famílias. As espécies foram ranqueadas através do índice proposto em três categorias de prioridade fitossociológica e socioeconômica (alta, média e baixa). A adequação da análise fatorial foi determinada pelos testes Bartlett e KMO. O teste de Bartlett avaliou a significância geral da matriz de correlação indicando que as correlações, em geral, são significantes ao nível de $1 \%$ de probabilidade. $O$ teste $\mathrm{KMO}$, indicou que as variáveis estão correlacionadas e o modelo fatorial apresentou um nível muito bom de adequação aos dados. Estes resultados respaldaram o emprego da análise fatorial para a extração de fatores e a estimação dos escores fatoriais. Os resultados foram estatisticamente validados para a construção do IFSE e, 25 espécies foram selecionadas como prioritárias (espécies-chave). Recomendou-se a densidade de plantio para essas espécies florestais na recuperação de áreas degradadas na Amazônia.

Palavras-chave: seleção de espécies arbóreas; análise multivariada; restauração florestal; espécie-chave.

\begin{abstract}
The identification of species influencing the occurrence of other associated species is of fundamental importance for the success of forest restoration of degraded areas. The objective of this work is to present a phytosociological and socioeconomic index (IFSE), obtained by techniques of factor analysis, modeling six quantitative variables (abundance, frequency, dominance, biomass, the commercial value of timber and amount of non-timber forest products) and equal number of qualitative variables, whose dummy variable is equal to one, assumed value for those species whose higher earnings amounted to $50 \%$ of the total for each quantitative variables analyzed. Sampling of the dense rain forest (407 ha) was composed by 80 plots of 0.25 ha $(4.91 \%$ of sampling intensity) and all individuals with diameter at $1.30 \mathrm{~m}$ above the ground $(\mathrm{DBH}) \geq 10 \mathrm{~cm}$ were identified and measured. It was recorded 10,105 individuals, including 493 species covering 58 families. The species were ranked by the index proposed in three categories of priority phytosociological and socioeconomic status (high, medium and low). The appropriateness of factor

1. Engenheiro Florestal, Dr., Pesquisador Associado do Museu Paraense Emílio Goeldi, Coordenação de Botânica, Av. Tancredo Neves, 1901, Caixa Postal 399, CEP 66040-170, Belém (PA). salomao@museu-goeldi.br

2. Engenheiro Agrônomo, Dr., Professor Associado da Universidade Federal Rural da Amazônia, Instituto Sócio-Ambiental e de Recursos Hídricos, Av. Tancredo Neves, 2501, CEP 66077-901, Belém (PA). antonio.santana@ufra.edu.br

3. Engenheiro Florestal, PhD., Pesquisador da Embrapa Amazônia Oriental (EMBRAPA/CPATU), Departamento de Floresta, Trav. Enéas Pinheiro, s/n, CEP 66095-100, Belém (PA). sbrienza@gmail.com

Recebido para publicação em 9/11/2010 e aceito em 24/10/2011
\end{abstract}


analysis was determined by tests of Bartlett and KMO. The Bartlett's test evaluated the overall significance of the correlation matrix and indicated that correlations were significant at $1 \%$ probability. The KMO test showed that the variables were correlated and the model of variance showed a very good level of fitness to the data. These results endorsed the use of factor analysis to extract factors and estimate the factor scores. The results were statistically validated for construction of the index IFSE and 25 species were selected as the priority ones (key species). And also, it was recomended the planting density for these forest species for restoration of degraded areas in Amazonia.

Keywords: selection of tree species; multivariate analysis; forest restoration; key species.

\section{INTRODUÇÃO}

A restauração ecológica é o processo de auxílio ao restabelecimento de um ecossistema que foi degradado, danificado ou destruído (SER, 2004) e que consiste de um conjunto de intervenções técnicas e científicas, de caráter intensivo, que visam garantir, no âmbito de uma metodologia técnicocientífica, a perenidade de um patrimônio natural. Geralmente o ecossistema que requer restauração foi transformado, danificado, degradado ou totalmente destruído como resultado direto ou indireto de atividades humanas. De acordo com Vieira et al. (2009) e Brienza Júnior et al. (1995) a degradação pode ser ambiental (ou ecológica) ou degradação da capacidade produtiva. A restauração ecológica envolve $\mathrm{o}$ atendimento a, pelo menos, nove atributos listados em Ser (2004). Dentre esses, destacamse que o ecossistema restaurado deve conter um conjunto característico de espécies (espécieschave) que ocorrem no ecossistema de referência, fornecendo uma estrutura apropriada de comunidade e, também, que seja constituído do maior número possível de espécies nativas. $\mathrm{Na}$ base da definição dos métodos adequados para a restauração ecológica dos ecossistemas duas questões são essenciais para o sucesso do objetivo, independentemente do método selecionado, qual espécie plantar e quanto plantar de cada espécie de modo a recobrir o solo e restaurar os processos ecológicos intrínsecos à vegetação em menos tempo, com menores perdas e menor custo.

Em áreas de mineração a céu aberto, em Porto Trombetas, na Amazônia, a bauxita é encontrada no subsolo, entre $5 \mathrm{~m}$ e $10 \mathrm{~m}$ de profundidade e em áreas geralmente cobertas pela floresta ombrófila densa. De acordo com a meta anual de produção, as áreas desmatadas (corte raso) variam de 400 ha a 500 ha por ano (Mineração Rio do Norte-MRN). Essas áreas mineradas não possuem mais resiliência sendo necessária, neste caso, a intervenção antrópica para a recuperação do ecossistema (RODRIGUES e GANDOLFI, 2001). Assim, após a lavra, tem início o processo de restauração da paisagem florestal via reflorestamento com espécies arbóreas majoritariamente nativas da Amazônia (SALOMÃO et al., 2002), concomitantemente a adição de solo superficial ("terra-preta") nas áreas de plantio para, entre outras, favorecer a sucessão natural (SALOMÃO et al., 2007). É oportuno ressaltar que o ecossistema artificial formado após a lavra pode ser classificado como de extrema degradação ambiental, pois as propriedades físicas, químicas e biológicas do solo foram profundamente alteradas, sendo superado somente pelos "lagos de rejeito" (FRANCO et al., 1992). A revegetação é parte essencial no processo de restauração de áreas degradadas, implicando na seleção de espécies adequadas ao plantio para acelerar os processos de recomposição florística da paisagem antropizada. Neste contexto, Moreira (2004) afirma que para o desenvolvimento de novas tecnologias e formas de manejo há que se intensificar pesquisas multidisciplinares para o sucesso da restauração florestal. Os estudos das espécies florestais nativas geralmente relacionam-se principalmente às características botânicas e dendrológicas (FARIA et al., 1997).

A identificação de espécies-chave, que são aquelas que controlam a estrutura da comunidade devido à sua abundância, distribuição espacial, biomassa, porte ou cobertura e que influenciam a ocorrência das demais espécies associadas, é de fundamental importância para o sucesso da restauração florestal em áreas degradadas, principalmente por mineração a céu aberto. Um importante instrumento da ecologia de comunidade é a análise multivariada, que trata todas as variáveis simultaneamente, sumariando os dados e revelando a sua estrutura com a menor perda de informações possível (GAUCH, 1982; PIELOU, 1984). Diferentemente da estatística clássica, que possibilita o teste de hipótese, as análises multivariadas se prestam mais a investigar os dados e gerar hipóteses (GAUCH， 1982; OLIVEIRA-FILHO， 1994). 
Recentemente as análises multivariadas tornaramse instrumentos acessíveis, apesar de há muito conhecidas (GOODALL, 1954).

Muitos estudos em comunidades vegetais utilizam como base esse tipo de análise, seja para agrupar amostras, classificar tipos de formações, relacionar variáveis ambientais a diferenças na comunidade ou mesmo para definir prioridades para a conservação (JOHNSON e WICHERN, 2007, OGUTU, 1996; KAPPELLE et al., 1995; TAGGART, 1994; LA ROI, 1992).

A análise multivariada foi empregada por Toledo et al. (2009) para caracterização fitossociológica em vegetação de cerrado no norte de Minas Gerais. Santos et al. (2007) analisaram a riqueza e a similaridade florística de fragmentos florestais no norte de Minas Gerais baseados no dendrograma e na distância euclidiana entre as parcelas, calculada a partir da matriz de correlação entre as variáveis selecionadas. Poucos estudos, no entanto, tratam simultaneamente vários tipos de formações vegetais por métodos de análise multivariada. Silva e Shepherd (1986) estabeleceram relações entre diferentes amostras de mata atlântica e floresta amazônica, utilizando a análise de agrupamento ao nível de gêneros. Compreender a dinâmica dos processos da floresta e visualizar as mudanças, detectar as causas e determinar quali e quantitativamente o conjunto das mesmas, constitui um grande desafio nos trabalhos de manejo de precisão para estabelecer relações e previsão de consequências que sejam ambientalmente corretas, economicamente viáveis e socialmente aceitas e justas (NAPPO, 2002).

Objetivou-se neste trabalho analisar a estrutura fitossociológica horizontal, a biomassa, o volume de madeira e seu respectivo valor comercial, além dos usos e utilidades (produtos florestais não madeireiros-PFNM) de espécies arbóreas da floresta primária densa, com base na geração de um índice fitossociológico e socioeconômico (IFSE) obtido por técnicas de análise multivariada, via hierarquização das espécies amostradas no inventário, em três categorias ecológicas e socioeconômicas previamente estabelecidas: prioridade de plantio alta, média e baixa. Concomitantemente à indicação das espécies de prioridade alta e média, foi sugerida a densidade de plantio das mesmas nas áreas de máxima antropização na Amazônia, ou seja, nas áreas de mineração a céu aberto cuja cobertura florestal primária foi suprimida, através de corte raso, para lavra do minério.

\section{MATERIAL E MÉTODO}

\section{Caracterização da área}

A área de estudo acha-se inserida na Região da Floresta Ombrófila Densa, Sub-região dos Baixos Platôs da Amazônia, domínio da floresta densa das baixas altitudes (submontana), cuja fisionomia refere-se à floresta localizada principalmente nos platôs terciários e terraços antigos e recentes, apresentando-se em dois estratos distintos: um emergente e outro uniforme. As principais espécies que caracterizam o estrato emergente são: Dinizia excelsa (angelim-pedra), Bertholletia excelsa (castanheira) e Cedrelinga catanaeformis (cedrorana). $\mathrm{O}$ estrato uniforme é caracterizado por Manilkara spp (maçarandubas), Protium spp (breus) e Pouteria spp (abius). Trata-se de florestas com alto volume de madeira de grande valor comercial. Comparada com outras áreas florestais da Amazônia é uma das mais imponentes, com sub-bosque limpo, boa regeneração natural e fácil penetração (RADAMBRASIL, 1974). O inventário florestal e fitossociológico foi realizado em uma área de 407 ha, no platô Almeidas $\left(01^{\circ} 45^{\prime} 16,0^{\prime \prime} \mathrm{S}\right.$ - 056 $\left.23^{\circ} 09,4^{\prime \prime} \mathrm{W}\right)$, com altitude média de $170 \mathrm{~m}$, localizado na Floresta Nacional Saracá-Taquera/ ICMBIo, no distrito de Porto Trombetas, município de Oriximiná, Pará, onde a Mineração Rio do Norte S.A. - MRN promove a lavra da bauxita, em vários platôs, desde 1979 (Figura 1).

\section{Processo de amostragem e variáveis analisadas}

$\mathrm{O}$ processo de amostragem utilizado no inventário florestal foi baseado na amostragem sistemática, também conhecida como seleção mecânica, onde as unidades amostrais são selecionadas segundo um rígido e pré-determinado esquema que procura cobrir toda a população. A vantagem mais importante da amostra sistemática, quando aplicada em florestas primárias, é que as unidades amostrais se distribuem mais uniformemente na população, originando uma maior representatividade, tornando-se eficiente para detectar tendência ou concentração de certas características, assim como é mais precisa na observação das variáveis tipológicas (CAMPOS e LEITE, 2002). A unidade amostral empregada tinha forma retangular com $10 \mathrm{~m}$ de largura por $250 \mathrm{~m}$ de comprimento $(0,25 \mathrm{ha})$, onde foram registradas, identificadas e tiveram mensurado o diâmetro a $1,30 \mathrm{~m}$ do solo (DAP) e estimada a altura do fuste de todas as árvores e palmeiras com DAP $\geq 10 \mathrm{~cm}$. 


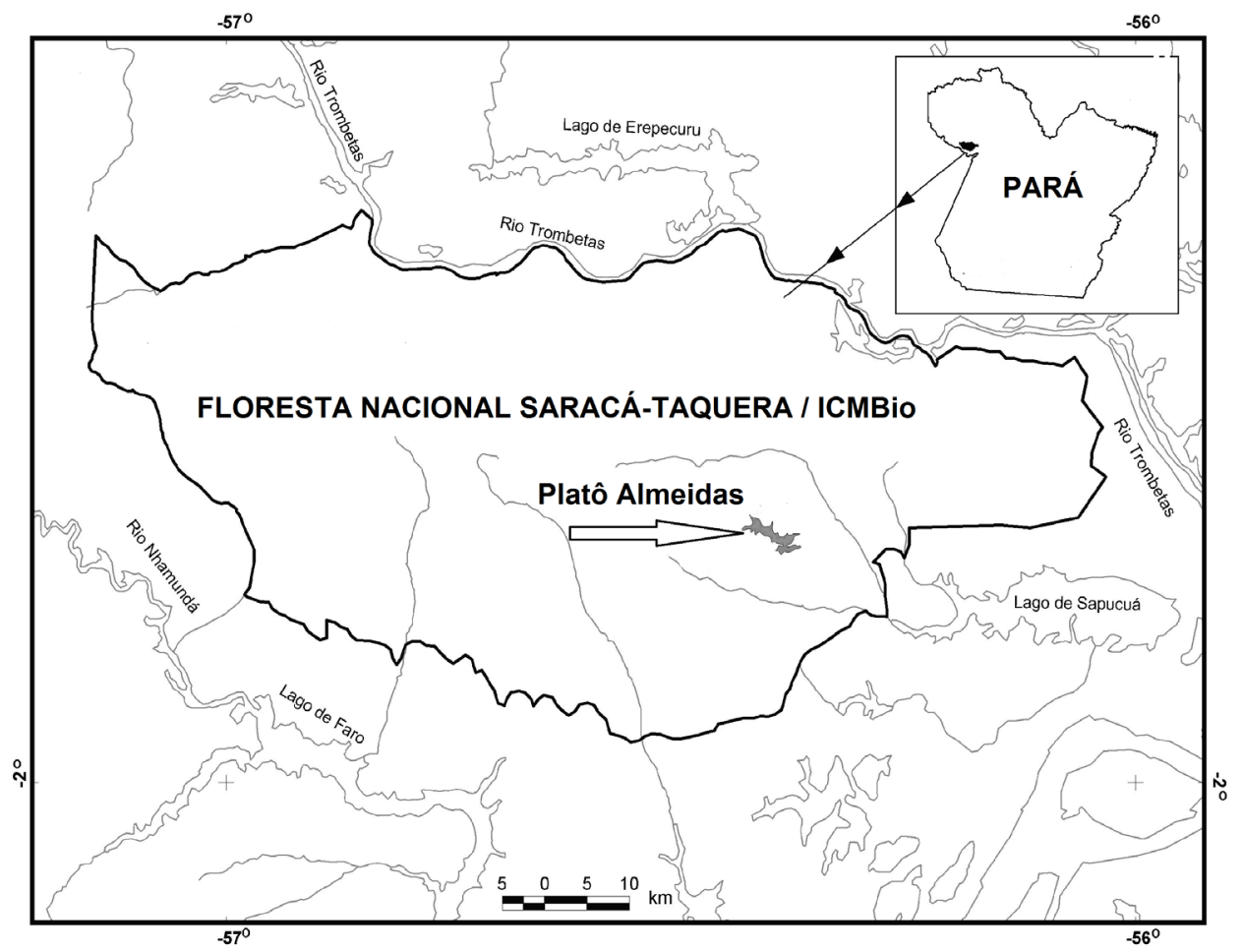

FIGURA 1: Localização da Floresta Nacional Sacará-Taquera/ICMBio, no Estado do Pará, onde se observa a conformação espacial do platô Almeidas.

FIGURE 1: Location of the National Forest Saracá-Taquera/ICMBio, state of Pará, where is the spatial conformation of Almeidas plateau.

A estrutura horizontal da floresta foi caracterizada através das variáveis fitossociológicas incluídas na análise fatorial como abundância (NI), frequência $(\mathrm{FQ})$ e área basal/dominância $(\mathrm{AB})$ das espécies registradas nas parcelas da amostra. Além destas variáveis, foram incluídas, na construção do índice, uma variável relativa à biomassa da espécie (Y), uma variável econômica relativa ao valor comercial da madeira em pé de cada espécie (VCM) e uma variável socioeconômica que abrangeu o uso e a utilidade expressado pelo número de produtos florestais não madeireiros da espécie (PFNM). A biomassa aérea viva (peso seco, em $\mathrm{kg}$ ) de todos os indivíduos das espécies foi estimada através da equação alométrica em função do diâmetro a $1,30 \mathrm{~m}$ do solo $(\mathrm{D}$, em $\mathrm{cm}), \mathrm{Y}=38.4908$ - 11.7883.D + 1.1926. $\mathrm{D}^{2}\left(\mathrm{r}^{2}=0,78\right)$, proposta por Brown et al. (1989). No caso da variável econômica (VCMr) multiplicou-se o volume comercial total $\left(\mathrm{m}^{3}\right)$ da espécie pelo respectivo valor da madeira em tora e em pé $\left(\mathrm{R} \$ / \mathrm{m}^{3}\right)$, de acordo com a Instrução Normativa $\mathrm{N}^{\circ}$ 02/2010, do Instituto de Desenvolvimento Florestal do Estado do Pará (IDEFLOR, 2010). A variável socioeconômica, referente ao uso/utilidade da espécie quantificou o número de aplicações
(PFNM) de cada espécie de acordo com Salomão et al. (1995), Shanley e Medina (2005), Shanley e Rosa (2005) e Salomão et al. (2007) em: AH (alimento para o homem), ME (espécie medicinal), CO (produção de corante), EA (produção de essência aromática), FI (produção de fibra), LA (produção de látex), $\mathrm{OE}$ (produção de óleos essenciais); $\mathrm{RE}$ (produção de resina) e VE (produção de substância venenosa).

\section{Modelo Analítico \\ Construção do índice fitossociológico e socioeconômico}

O modelo do índice fitossociológico e socioeconômico (IFSE), proposto neste trabalho, é uma função de seis variáveis quantitativas e igual número de variáveis qualitativas expressadas pelas respectivas variáveis dummy (VD).

$I F S E=f(N I r, N I r-V D, F Q r, F Q r-V D, A B r$, $A B r-V D, Y r, Y r-V D, V C M r, V C M r-V D, P F N M r$, $P F N M r-V D$ )

onde: abundância relativa (NIr), frequência absoluta (FQ), área basal / dominância relativa $(\mathrm{ABr})$, biomassa relativa (Yr), valor comercial da madeira em tora e em pé relativa (VCMr), 
quantidade de produtos florestais não madeireiros relativa (PFNMr).

Variáveis quantitativas: $\mathrm{NIr}=\left(\mathrm{n}^{\mathrm{o}}\right.$ de indivíduos da espécie / $\mathrm{n}^{\circ}$ total de indivíduos) * $100 ; \mathrm{FQr}=\left(\mathrm{n}^{\mathrm{o}}\right.$ de parcelas de ocorrência da espécie / soma do $\mathrm{n}^{\circ}$ total de parcelas de ocorrência de todas as espécies) ${ }_{*} 100 ; \mathrm{ABr}=$ (área basal total dos indivíduos da espécie / área seccional total de todos os indivíduos das espécies) $* 100 ; \mathrm{Yr}=($ biomassa aérea viva em pé da espécie / biomassa aérea viva em pé de todas as espécie) ${ }_{*} 100 ; \mathrm{VCMr}=($ valor comercial da madeira em tora e em pé da espécie / valor comercial da madeira em tora e em pé de todas as espécies) ${ }_{*} 100 ; \mathrm{PFNMr}=$ (quantidade de usos ou utilidades da espécie / soma das quantidade de usos ou utilidades de todas as espécies) ${ }_{*} 100$.

Variáveis qualitativas: os fenômenos analisados, cuja variável qualitativa ou dummy assumiu valor igual a 1 , referem-se àquelas espécies cujos maiores valores acumulados da abundância, frequência, área basal, biomassa, valor comercial da madeira e número de produtos florestais não madeireiros responderam por mais de $50 \%$ do valor total de qualqueruma destas variáveis. Considerandose, por exemplo, a variável abundância procedeuse da seguinte forma: i) após a hierarquização decrescente da abundância das espécies (do maior para o menor valor da variável) efetuou-se a soma do número total de indivíduos de cada espécie até se atingir $50 \%$ do total de indivíduos de todas as espécies, as espécies aí incluídas receberam valor 1 na variável dummy, enquanto as demais o valor zero; e ii) raciocínio análogo foi feito para todas as demais variáveis. Normalmente essas variáveis constituem um número pequeno em relação ao tamanho da amostra. Posteriormente, foram relativizadas, ou seja, os valores foram expressos em percentagem cuja soma perfazia $100 \%$.

\section{Análise fatorial}

A análise fatorial, de modo geral, é utilizada para analisar a estrutura das inter-relações (correlações) entre um grande número de variáveis quantitativas e/ou qualitativas para definir um conjunto de dimensões latentes comuns, com a missão de facilitar a compreensão sobre a estrutura e representação da nuvem de dados, chamadas de fatores comuns. Com o emprego dessa técnica, inicialmente pode-se identificar as dimensões isoladas da estrutura dos dados e então determinar o grau em que cada variável é explicada por cada dimensão latente ou fator. Depois dessa etapa, a análise fatorial pode ser empregada para reduzir o número de variáveis e, mesmo assim, representar adequadamente a massa de dados (MINGOTI, 2005; SANTANA, 2007). O modelo geral de análise fatorial pode ser apresentado na forma matricial como em Dillon e Goldstein (1984):

$$
X=\alpha F+\varepsilon
$$

em que: (i) $X=$ é o p-dimensional vetor transposto das variáveis observáveis, denotado por $X=\left(x_{1}, x_{2}, \ldots, x_{p}\right)^{\prime}$; (ii) $F=$ é o q-dimensional vetor transposto de variáveis não observáveis ou variáveis latentes chamadas de fatores comuns, denotado por, $F=\left(f_{l}, f_{2}, \ldots, f_{q}\right)^{\prime}$, sendo que $\mathrm{q}<\mathrm{p}$; (iii) $\varepsilon=$ é o p-dimensional vetor transposto de variáveis aleatórias ou fatores únicos, $\mathrm{e}=\left(e_{1}, e_{2}, \ldots, e_{p}\right)^{\prime} \mathrm{e}$, (iv) $\alpha=$ é a matriz $(\mathrm{p}, \mathrm{q})$ de constantes desconhecidas, chamadas de cargas fatoriais.

Para a definição do índice fitossociológico e socioeconômico com variável dummy (IFSE), procedeu-se, conforme sugerido por Santana (2007), a estimação da matriz de escores fatoriais após a rotação ortogonal da estrutura fatorial inicial. $\mathrm{O}$ escore fatorial, por definição, situa cada observação no espaço dos fatores comuns. Para cada fator $f_{j}$, o i-ésimo escore fatorial extraído é definido por $F_{i j}$, expresso por meio de uma combinação linear da forma descrita em Dillon e Goldstein (1984):

$$
F_{i j}=b_{I} x_{i 1}+b_{2} x_{i 2}+\ldots+b_{p} x_{i p} ;(i=1,2, \ldots, n ; j=
$$

em que: $b_{i}$ são os coeficientes de regressão estimados para os $n$ escores fatoriais comuns; $x_{i j}$ são as $n$ observações das $p$ variáveis observáveis, padronizadas pelo método Z-score para apresentar uma distribuição com média 0 e desvio igual a 1. A variável $F_{i j}$ não é observável, mas pode ser estimada por meio das técnicas de análise fatorial, utilizandose a matriz de observações do vetor $x$ de variáveis observáveis.

Para a construção do IFSE, o escore fatorial $(F)$ foi padronizado para se obter valores positivos dos escores originais para variar entre 0 e $1(F P$ ) e permitir a hierarquização das espécies. A fórmula matemática é a seguinte:

$$
F P_{i}=\left(\frac{F_{i}-F_{\text {min }}}{F_{\max }-F_{\min }}\right)
$$

Em que, Fmáx e Fmín são os valores máximo e mínimo observados para os escores fatoriais associados às variáveis (abundância, frequência, área basal, valor comercial da madeira, biomassa, número de PFNM) das espécies. 
O IFSE foi definido como uma combinação linear desses escores fatoriais e a proporção da variância explicada por cada fator, em relação à variância explicada pelos fatores comuns, com a variável dummy $(k)$. A expressão matemática é dada por (SANTANA, 2007):

$$
I F S E_{i, k}=\sum_{j-1}^{q}\left(\frac{\lambda_{j}}{\sum_{j} \lambda_{j}} F P_{i j}\right)
$$

Em que, $\lambda$ é a variância explicada por cada fator e $\Sigma \lambda$ é a soma total da variância explicada pelo conjunto de fatores comuns extraídos.

Para facilitar a interpretação dos resultados, foram estabelecidos três intervalos de valores do IFSE; obtidos pela diferença entre o maior e o menor valor calculado, dividida por 3 ; aqui denominada amplitude de ranqueamento $(A R)$. Espécies de alta prioridade foram aquelas com valores situados no intervalo entre o maior valor do IFSE, menos o valor da amplitude de ranqueamento; espécies com prioridade baixa foram aquelas cujos índices calculados encontravam-se no intervalo entre $o$ menor valor do IFSE, mais o valor da amplitude de ranqueamento. As espécies de prioridade média foram aquelas situadas no intervalo entre o limite inferior das de prioridade alta e o limite superior das de prioridade baixa.

Para aferir a adequação do método à amostra de dados foram aplicados os dois principais testes: teste de Kaiser-Meyer-Olkin (KMO) que se baseia no princípio de que a inversa da matriz de correlação se aproxima da matriz diagonal - para tanto compara as correlações entre as variáveis observáveis (HAIR et al., 2005) e, teste de esfericidade de Bartlett, que avalia a significância geral da matriz de correlação, ou seja, testa a hipótese nula de que a matriz de correlação é uma matriz identidade. Os resultados foram obtidos por meio do software SPSS Statistics $17.0^{\mathrm{TM}}$.

\section{RESULTADOS E DISCUSSÃO}

Foram registrados, nas 80 parcelas $(0,25$ ha cada, totalizando 20 ha) do inventário florestal do platô Almeidas, na Floresta Nacional SaracáTaquera/ICMBio, 10.105 indivíduos (DAP $\geq$ $10 \mathrm{~cm}$ ), totalizando 493 espécies, distribuídas em 58 famílias; foram computados à parte 34 cipós de cinco espécies.

\section{Índice fitossociológico e socioeconômico - IFSE}

No presente estudo a adequação da análise fatorial foi determinada pelos testes Bartlett e KMO. O teste de Bartlett avaliou a significância geral da matriz de correlação e apresentou um valor de 7.444,906, indicando que as correlações, em geral, são significantes ao nível de $1 \%$ de probabilidade, ou seja, a amostra dos dados pode ser submetida à análise fatorial. O teste $\mathrm{KMO}$, da ordem de 0,72 indicou que as variáveis estão correlacionadas e o modelo fatorial apresentou um nível bom de adequação aos dados. Estes resultados respaldam o emprego da análise fatorial para a extração de fatores e a estimação dos escores fatoriais. Foram extraídos três fatores que, juntos, explicaram 78,187 \% da variância total da nuvem de dados e em termos individuais o fator 1 explicou $34,895 \%$ da variância total, o fator 2 explicou $28,547 \%$ e o fator 3 explicou 14,745 \% (Tabela 1). A comunalidade, que representa o grau em que a variância de cada variável é explicada pelos fatores comuns, foi superior a 0,65 , indicando que pelo menos $65 \%$ da variância de cada variável foram explicados pelos três fatores comuns. Isto significa que a variância única é reduzida e o modelo é adequado para expressar o fenômeno estudado por meio das dimensões latentes extraídas do modelo.

$\mathrm{O}$ fator 1 pode ser compreendido como fator econômico das espécies em se considerando que o porte de uma árvore é uma função direta do diâmetro do tronco e da altura. Observou-se que a área basal e a biomassa, que são uma função do diâmetro, e o valor comercial da madeira, que é uma função do diâmetro e da altura, estão inseridos nesta mesma dimensão. Este fator, considerado o mais importante, responde por quase $35 \%$ da variância explicada. O fator 2 pode ser denominado como fator ecológico das espécies uma vez que envolveu a abundância, que informa o número de indivíduos da espécie por unidade de área, e a frequência, que relaciona a distribuição espacial dos indivíduos da espécie na área amostrada. Esta dimensão respondeu por cerca de aproximadamente $29 \%$ do total da variância explicada. O fator 3 pode ser entendido como fator social das espécies, uma vez que abrangeu os usos e utilidades das mesmas, entendidos no modelo como sendo o número de produtos florestais não madeireiros intrínsecos a cada espécie. Este fator foi responsável por aproximadamente $15 \%$ da variância total explicada.

O índice fitossociológico e socioeconômico foi construído para $78,187 \%$ da variância total 
TABELA 1: Matriz de cargas fatoriais ( $\alpha$ ) rotacionadas após a rotação ortogonal pelo método Varimax com respectivas variáveis dos componentes obtidos.

TABLE 1: Matrix of factor loadings $(\alpha)$ rotated after the orthogonal rotation method Varimax with their component variables obtained.

\begin{tabular}{lcccc}
\hline \multirow{2}{*}{ Variável } & \multicolumn{3}{c}{ Componente } & \multirow{2}{*}{ Comunalidade } \\
\cline { 2 - 4 } & 1 & 2 & 3 & 0,651 \\
Abundância relativa & 0,182 & $\mathbf{0 , 7 7 1}$ & 0,154 & 0,050 \\
Abundância relativa variável dummy & 0,055 & $\mathbf{0 , 8 8 1}$ & 0,050 & 0,782 \\
Frequência relativa & 0,378 & $\mathbf{0 , 7 9 9}$ & 0,092 & 0,790 \\
Frequência relativa variável dummy & 0,145 & $\mathbf{0 , 8 6 1}$ & 0,021 & 0,763 \\
Área basal relativa & $\mathbf{0 , 7 4 3}$ & 0,478 & 0,201 & 0,822 \\
Área basal relativa variável dummy & $\mathbf{0 , 7 9 2}$ & 0,435 & 0,055 & 0,820 \\
Biomassa relativa & $\mathbf{0 , 7 8 4}$ & 0,352 & 0,192 & 0,776 \\
Biomassa relativa variável dummy & $\mathbf{0 , 8 4 1}$ & 0,329 & 0,058 & 0,819 \\
Valor comercial madeira & $\mathbf{0 , 8 4 4}$ & $-0,011$ & 0,075 & 0,718 \\
Valor comercial madeira variável dummy & $\mathbf{0 , 8 6 6}$ & $-0,040$ & $-0,013$ & 0,752 \\
Produto florestal não madeireiro & 0,042 & 0,055 & $\mathbf{0 , 9 2 3}$ & 0,857 \\
Produto florestal não madeireiro variável dummy & 0,152 & 0,138 & $\mathbf{0 , 8 9 1}$ & 0,835 \\
\hline Autovalor & 4,187 & 3,426 & 1,769 & 9,382 \\
Variância explicada (\%) & 34,895 & 28,547 & 14,745 & 78,187 \\
\hline
\end{tabular}

explicada pelos três fatores extraídos. Em função da significância estatística da análise e a forte aderência aos aspectos teóricos e práticos refletido nas dimensões fatoriais econômica, ambiental e social, o IFSE pode se revelar como importante indicador para a escolha das espécies com maior potencial de reproduzir essas características nos processos de restauração florestal de áreas degradadas. O índice para esta solução mostrou que $78,187 \%$ da variância total são representados pela informação contida na matriz fatorial da solução em termos dos três fatores. O índice é considerado muito bom, e as variáveis estão, como esperado, estreitamente relacionadas umas com as outras.

O IFSE calculado variou de $0,164 \%$ a $0,752 \%$ consequentemente, a amplitude total de $0,588 \%$, dividida por 3 , resultou em uma amplitude de ranqueamento (AR) de $0,196 \%$, que gerou os três intervalos de categorias pré-estabelecidas quanto à prioridade ecológica e socioeconômica das espécies em alta $(0,752 \%$ a $0,556 \%)$, média $(0,555 \%$ a $0,361 \%)$ e baixa $(0,360 \%$ a $0,164 \%)$. Foram classificadas três espécies como de alta prioridade, 22 como de prioridade média e as demais 468 espécies como de baixa prioridade. A relação das espécies classificadas como de alta e média prioridades é apresentada na Tabela 2 .

\section{Seleção de espécies pelo IFSE}

A seleção de espécies arbóreas a ser usada sob diferentes métodos de restauração é decisiva quando o objetivo é o de procurar aproximar-se ao máximo da composição florística e estrutura vertical da floresta primitiva anteriormente existente na área (RODRIGUES e GANDOLFI, 2004). A escolha das espécies deve ser fundamentada em pesquisas desenvolvidas na mesma área a ser restaurada ou em outras similares. A longo prazo, devem ser considerados vários aspectos para o sucesso da restauração, entre eles, aqueles que procuram garantir a variabilidade genética (MCKAY et al., 2005). Neste estudo, em que foram selecionadas 25 espécies com prioridade alta e média, considerando-se algumas variáveis fitossociológicas e socioeconômicas incluídas no índice, houve uma pesquisa de campo intensiva através do inventário florestal e fitossociológico com 4,91\% de intensidade amostral e erro amostral de 3,14\% para probabilidade $t$-student a $95 \%$. Como se comportaram essas espécies selecionadas em relação às variáveis incluídas na construção do índice?

A abundância informa quantos indivíduos de diferentes espécies existem por unidade de área. No presente estudo, somando-se as maiores abundâncias observou-se que apenas 28 espécies (ou $5,7 \%$ do total) responderam por mais de $50 \%$ do total de 10.105 indivíduos, fato também observado por Salomão et al. (2007) nas florestas do médio Rio Xingu (PA). Essas espécies foram discriminadas no modelo com uma variável qualitativa de valor 1 por serem aquelas de maior importância em relação 
TABELA 2: Relação das espécies-chave classificadas nas categorias ecológicas e socioeconômicas como de prioridade alta (1) e média (2), determinada através do índice fitossociológico e socioeconômico (IFSE); da floresta ombrófila densa, platô Almeidas, Flona Saracá-Taquera/ ICMBio, Porto Trombetas, Pará.

TABLE 2: List of key species, categorized as ecological and socioeconomic of high priority (1) and average (2), determined by the socioeconomic and phytosociological index (IFSE) of dense rain forest, plateau Almeidas Saracá-Taquera/ICMBio National Forest, Puerto Trumpets, Pará state.

\begin{tabular}{|c|c|c|c|c|c|c|c|c|c|}
\hline Nome científico & Família & $\begin{array}{l}\text { NIr } \\
(\%)\end{array}$ & $\begin{array}{l}\text { FQr } \\
(\%)\end{array}$ & $\begin{array}{l}\mathrm{ABr} \\
(\%)\end{array}$ & $\begin{array}{c}\mathrm{Yr} \\
(\%)\end{array}$ & $\begin{array}{c}\mathrm{VCMr} \\
(\%)\end{array}$ & $\begin{array}{c}\text { PFNMr } \\
(\%)\end{array}$ & $\begin{array}{c}\text { IFSE } \\
(\%)\end{array}$ & CAT \\
\hline Oenocarpus bacaba & Arecaceae & 12,95 & 1,61 & 4,62 & 3,25 & 0,00 & 1,75 & 0,752 & 1 \\
\hline Geissopermum sericeum & Apocynaceae & 3,73 & 1,53 & 4,14 & 4,03 & 2,86 & 0,88 & 0,697 & 1 \\
\hline Bertholletia excelsa & Lecythidaceae & 0,27 & 0,40 & 5,90 & 7,37 & 0,00 & 1,75 & 0,653 & 1 \\
\hline Pouteria oppositifolia & Sapotaceae & 0,60 & 0,68 & 3,27 & 3,77 & 8,47 & 0,00 & 0,552 & 2 \\
\hline Tetragastris panamensis & Burseraceae & 2,52 & 1,47 & 1,57 & 1,34 & 1,18 & 0,88 & 0,520 & 2 \\
\hline Aniba canelila & Lauraceae & 0,39 & 0,50 & 1,31 & 1,46 & 3,29 & 1,75 & 0,514 & 2 \\
\hline Goupia glabra & Celastraceae & 0,49 & 0,64 & 1,54 & 1,75 & 1,94 & 0,88 & 0,479 & 2 \\
\hline Bellucia grossularioides & Melastomataceae & 1,19 & 0,72 & 0,96 & 0,87 & 0,00 & 1,75 & 0,455 & 2 \\
\hline Hevea guianensis & Euphorbiaceae & 1,15 & 1,00 & 2,28 & 2,42 & 0,00 & 0,88 & 0,455 & 2 \\
\hline Licaria rigida & Lauraceae & 1,24 & 1,11 & 1,48 & 1,44 & 2,49 & 0,00 & 0,452 & 2 \\
\hline Dipteryx odorata & Fabaceae & 0,14 & 0,22 & 0,88 & 1,05 & 2,85 & 0,88 & 0,447 & 2 \\
\hline Aspidosperma nitidum & Apocynaceae & 0,36 & 0,64 & 2,16 & 2,55 & 2,89 & 0,00 & 0,428 & 2 \\
\hline Brosimum guianense & Moraceae & 0,86 & 1,06 & 1,29 & 1,32 & 1,41 & 0,00 & 0,427 & 2 \\
\hline Virola michelli & Myristicaceae & 0,87 & 1,00 & 0,74 & 0,68 & 0,81 & 0,88 & 0,415 & 2 \\
\hline Swartzia recurva & Fabaceae & 0,59 & 0,80 & 1,61 & 1,78 & 1,60 & 0,00 & 0,414 & 2 \\
\hline Endopleura uchi & Humiriaceae & 0,58 & 0,72 & 1,76 & 1,94 & 2,09 & 0,00 & 0,400 & 2 \\
\hline Brosimum rubescens & Moraceae & 0,52 & 0,76 & 1,72 & 1,90 & 2,03 & 0,00 & 0,398 & 2 \\
\hline Chimarrhis turbinata & Rubiaceae & 0,35 & 0,58 & 1,64 & 1,90 & 2,43 & 0,00 & 0,395 & 2 \\
\hline Protium crenata & Burseraceae & 0,45 & 0,28 & 0,90 & 0,95 & 1,00 & 0,88 & 0,395 & 2 \\
\hline Protium tenuifolium & Burseraceae & 1,04 & 1,13 & 0,73 & 0,65 & 0,61 & 0,88 & 0,381 & 2 \\
\hline Protium hebetatum & Burseraceae & 1,09 & 1,08 & 0,43 & 0,32 & 0,33 & 0,88 & 0,379 & 2 \\
\hline Rinorea riana & Violaceae & 4,86 & 1,33 & 1,04 & 0,60 & 0,00 & 0,00 & 0,367 & 2 \\
\hline Eschweilera coriacea & Lecythidaceae & 0,82 & 0,82 & 0,88 & 0,87 & 0,96 & 0,00 & 0,364 & 2 \\
\hline Pouteria gongrypii & Sapotaceae & 1,23 & 0,98 & 1,00 & 0,90 & 0,00 & 0,00 & 0,364 & 2 \\
\hline Lecythis prancei & Lecythidaceae & 0,56 & 0,78 & 1,01 & 1,04 & 1,61 & 0,00 & 0,361 & 2 \\
\hline Totais & & 38,9 & 21,88 & 44,85 & 46,14 & 40,87 & 14,91 & 11,46 & - \\
\hline
\end{tabular}

Em que: abundância relativa (NIr), variável dummy (VD), frequência relativa (FQr), área basal relativa (ABr), biomassa relativa (Yr), valor comercial da madeira em pé relativa (VCMr), quantidade de produtos florestais não madeireiros relativa (PFNMr), categoria de prioridade fitossociológica e socioeconômica (CAT), CAT = 1 - prioridade alta, CAT $=2$ - prioridade média.

à abundância. Entre as 25 espécies selecionadas pelo IFSE, 13 apresentaram esta qualidade com variável dummy (VD) igual à unidade. Também, a abundância relativa dessas 25 espécies selecionadas equivaleu a praticamente $39 \%$ da abundância de todas as 493 espécies amostradas (Tabela 2).

A frequência permite conhecer a distribuição espacial da espécie na área estudada. A palmeira Oenocarpus bacaba ocorreu em todas as 80 parcelas, podendo-se concluir que a espécie possui distribuição uniforme (OLIVEIRA-FILHO e RATTER, 1995) em toda a área do platô. Em contraposição, outras 97 espécies $(19,7 \quad \%$ do total) ocorreram em apenas uma unidade amostral; com certeza essas espécies possuem distribuição aleatória (OLIVEIRA-FILHO e RATTER, 1995). $\mathrm{Na}$ amostragem, 20 espécies ocorreram em mais da metade do total de parcelas (variável dummy igual a 1), evidenciando uma tendência a uma distribuição uniforme; dentre estas, 13 foram incluídas entre as 25 selecionadas através do IFSE (Tabela 2).

A dominância é aqui entendida como sendo a área seccional do tronco a $1,30 \mathrm{~m}$ do solo. $\mathrm{O}$ somatório dessas áreas seccionais dos indivíduos 
da espécie, por unidade de área física, é traduzido pela área basal que é uma importante ferramenta para compreensão da floresta como um todo e de suas populações. Apenas 30 espécies (ou 6,1\%) responderam por mais de $50 \%$ da área basal total. A seleção, através do IFSE (Tabela 2), indicou 23 destas espécies, que apresentaram uma área basal equivalente a $44,9 \%$ do total.

$\mathrm{O}$ valor da biomassa aérea viva das árvores de uma floresta é majoritariamente representado pelo resultado de um pequeno número de árvores de maiores diâmetros ou de um grande número de indivíduos de menores diâmetros. Entre as 493 espécies amostradas, 27 apresentaram valor cumulativo de biomassa superior a $50 \%$ do total, sendo que o IFSE conseguiu captar 21 dessas espécies (Tabela 2). As 25 espécies selecionadas pelo índice tiveram uma participação relativa de $46,1 \%$ do valor da biomassa para totalidade das espécies.

Dentre as 493 espécies amostradas, 292 apresentaram valor comercial da madeira em tora e em pé, sendo que apenas 21 delas apresentaram volumes com valores cumulativos superiores a $50 \%$ do total. A seleção, através do IFSE, conseguiu incluir 13 dessas espécies $(\mathrm{VD}=1)$, que representaram, em conjunto, um volume comercial equivalente a $40,9 \%$ do total (Tabela 2). Considerando aquelas espécies que foram excluídas pelo índice como Cordia bicolor (freijó-branco), Hymenolobium nitidum (angelimpedra folha-grande), Tabebuia serratifolia (ipêamarelo), Lecythis prancei (jarana-amarela), Manilkara amazonica (maparajuba folha-miúda), Hymenolobium flavum (angelim-fava-amarela), Dinizia excelsa (angelim-vermelho) e Astronium gracile (muiracatiara), sugere-se que, por se tratar de espécies de alto ou médio valor comercial podem, e devem ser também priorizadas nos trabalhos de restauração florestal (GUIMARÃES NETO et al. 2004, RODRIGUES e GANDOLFI, 2001).

Os produtos florestais não madeireiros (PFNM) são de muita importância, sobretudo para as populações tradicionais da Amazônia (SHANLEY e MEDINA, 2005; SHANLEY e ROSA, 2005) no que concerne a seleção de espécies para restauração florestal, daí terem sido incluídas no modelo. Esta variável foi analisada quanto ao número de PFNM que cada espécie apresentava. Uma centena de espécies apresentaram usos e utilidades entre as 493 amostradas, sendo que entre aquelas apenas 12 foram incluídas pelo índice entre as 25 indicadas como de prioridade alta e média (Tabela 2). Oportuno se faz esclarecer que 14 espécies, entre aquelas 100, apresentaram mais de um PFNM, sendo que quatro delas foram captadas pelo modelo - Aniba canelila (casca-preciosa), Oenocarpus bacaba (bacaba), Bertholletia excelsa (castanheira) e Bellucia grossularioides (muúba).

Em síntese, as variáveis quantitativas e qualitativas incluídas no índice e que envolveram fatores ecológicos, econômicos e sociais permitiram definir 25 espécies-chave para o sucesso da restauração florestal sem, no entanto, excluir a opção de inclusão de outras espécies, uma vez que as legislações estaduais de alguns estados brasileiros estabeleceram um mínimo de 80 espécies nos trabalhos com este objetivo (São Paulo: Resolução SMA 47, de 21/11/2003 e Pará: Decreto no 31.594, de 27/01/2010).

Os plantios para recuperação de áreas degradadas na Amazônia, implantados nas áreas de extração mineral, têm utilizado majoritariamente espécies heliófilas, como as leguminosas, pela facilidade de fixação biológica do nitrogênio (NAPPO, 2002; BACHA e BARROS, 2004; MOLINARO, 2005). Stella (2002) testou uma metodologia de seleção de espécies arbóreas tropicais prioritárias para a conservação, a partir de um total de 214 espécies lenhosas conhecidas, sendo cinco delas selecionadas. A análise do sistema dos algoritmos, bem como a da seleção e uso dos critérios revelou pontos fortes e fracos da metodologia, tendo sido concluído que a metodologia pode ser útil para a seleção de espécies arbóreas tropicais para diferentes fins.

Segundo Lamb et al. (2005), alguns outros aspectos devem ser considerados durante a escolha de espécies, tais como: (i) espécies vegetais de crescimento rápido que excluam ervas daninhas e gramíneas agressivas; (ii) espécies com baixa dispersão natural (por exemplo, de sementes grandes); (iii) espécies que ofereçam alimentação, proteção e possibilidade de aninhar à fauna residente; (iv) espécies raras e ameaçadas de extinção. Certos cuidados devem ser levados em consideração quando se opta pelo o uso de espécies exóticas. Essas plantas devem ser usadas em locais com degradação extrema e onde sua reprodução possa ser controlada de forma a prever sua erradicação de forma rápida, caso necessário.

Salomão et al. (2002) avaliaram o crescimento anual do diâmetro basal médio de 35 espécies empregadas pela MRN em suas 
áreas anuais de restauração florestal pós-lavra, extremamente degradadas. Foi calculado o incremento periódico anual (IPA) da espécie, relativo ao crescimento do diâmetro basal de cada indivíduo, gerando-se, a seguir o IPA médio da espécie. Quatro espécies foram consideradas como excelentes quanto à aptidão ecológica por apresentarem incremento anual superior ao dobro da média geral (Senna multijuga, Stryphnodendron guianensis, Sclerolobium paniculata e Tapirira guianensis); seis espécies foram classificadas como boas (Acacia polyphylla, Anacardium occidentale, Parkia multijuga, Inga edulis, Eugenia cumini e Abarema turbinata); outros $50 \%$ do total de espécies analisadas foram consideradas com aptidão regular e sete espécies foram enquadradas como de fraca aptidão.

\section{Densidade de Plantio}

O método de plantio adensado, onde são plantadas mais de mil mudas por hectare, é indicado para a restauração de áreas de reserva legal e de preservação permanente que originalmente eram recobertas pela floresta ombrófila e que foram degradadas, principalmente nas áreas utilizadas pela mineração a céu aberto. As áreas com explotação mineral apresentam alta degradação, precisando, portanto, de práticas especiais para sua restauração. Dada a alta rentabilidade da atividade minerária e as relativas pequenas extensões espaciais das áreas de lavra geralmente, os recursos financeiros são mais expressivos para a restauração florestal. Por norma, em alguns países, a lei obriga as empresas a restaurar as áreas após sua explotação, entretanto, algumas se limitam a recuperar as áreas perturbadas, em alguns casos com espécies exóticas, não restaurando, portanto, o habitat natural (LAMB, 1994). Se a finalidade é realmente a restauração da área degradada, o primeiro esforço deve ser direcionado para a recuperação do solo e de sua fertilidade para, posteriormente, continuar com as demais etapas do processo. A melhor prática para a restauração dessas áreas consiste em reutilizar as camadas superficiais do solo que foram retiradas e armazenadas enquanto ocorria a explotação do subsolo (PARROTA e KNOWLES, 2001; SALOMÃO et al. 2002). Por sua vez, é ideal a reconfiguração das características topográficas da área, de forma tal que seja minimizada a erosão e restabelecida a drenagem. Uma vez que as condições do solo para plantio são recuperadas, a restauração pode ser realizada por meio de várias técnicas, sendo a mais comum o plantio adensado. Nos trabalhos de restauração florestal, nas áreas anuais pós-lavra, desenvolvidos pela MRN, desde 1981, em Porto Trombetas (PA), houve variações nos adensamentos empregados assim como na origem (exóticas, nativas e introduzidas) e quantidade de espécies utilizadas (SALOMÃO et al. 2002). Atualmente, são plantadas 1.667 mudas ha $^{-1}$ (espaçamento de $3 \mathrm{~m}$ na linha e de $2 \mathrm{~m}$ entre linhas de plantio) em cerca de 400-500 ha anuais.

Considerando-se, então, a seleção das espécies-chave obtida através do IFSE proposto, o próximo passo consiste na quantificação do número de mudas a ser plantado de cada espécie nas áreas anuais de restauração florestal. Através do inventário florestal e fitossociológico executado antes da supressão da vegetação (corte raso) para lavra da bauxita, obteve-se as estimativas da abundância absoluta e relativa de cada uma 493 espécies registradas. De posse dessas informações foi construída a Tabela 3,indicando o número de mudas a plantar de cada espécie-chave partindo-se da premissa que: i) o percentual de mudas relativo às espécies selecionadas seria $10 \%$ superior àquele registrado no inventário, ii) nenhuma espécie deveria exceder a $20 \%$ do total de mudas plantadas por unidade de área e, iii) quando o número de mudas calculado de uma determinada espécie fosse inferior a 10 mudas ha $^{-1}$ ele seria arredondado para este limite, gerando o número de mudas efetivo a plantar da espécie. Observou-se que no caso do platô Almeidas, entre as 25 espécies selecionadas pelo IFSE, quatro apresentaram número calculado de mudas a plantar inferior a 10 mudas ha $^{-1}$ (Tabela 3). O número de mudas efetivo a plantar das 25 espécies foi de 736, ou seja, 44,2 \% do total de 1.667 mudas ha ${ }^{-1}$ previstas para a restauração florestal. As demais 931 mudas $\mathrm{ha}^{-1}$ devem ser complementadas por no mínimo 55 outras espécies, entre as demais 468 que foram registradas no inventário. Adicionalmente, pode-se optar pelas espécies prioritárias para a conservação relacionadas nas listas oficiais do Ibama e da Sema/PA e que se encontram em risco de extinção. Uma outra estratégia seria incrementar os plantios priorizando as espécies de valor comercial da madeira que ocorriam no platô, como aquelas já citadas anteriormente e, também, aquelas que apresentam usos e utilidades de seus produtos florestais não madeireiros (SHANLEY e MEDINA, 2005; SHANLEY e ROSA, 2005). Outra opção interessante seria complementar o número de espécies projetado (1.667 mudas $\left.\mathrm{ha}^{-1}\right)$ com aquelas classificadas pelo IFSE a partir da $25^{\mathrm{a}}$ posição, ou 
seja, as de categoria 3 (baixa prioridade) e com maiores valores do índice. Adicionalmente, há de se considerar a proporção desejada de $40 \%$ do total de espécies de qualquer um dos grupos ecológicos: pioneiras e climácicas (GUIMARÃES NETO et al., 2004; GAMA-RODRIGUES et al., 2008).

Ao se procurar caracterizar as espécieschave de um determinado ambiente, não se pode excluir da análise as demais, pois, como se sabe, cerca de $1 /{ }_{6}$ a $1 / 3$ das espécies ocorrem com apenas um indivíduo na totalidade dos estudos desenvolvidos em florestas primárias na Amazônia (SALOMÃO et al., 2007). Neste trabalho, foi observada a ocorrência de 88 espécies (ou 17,9\% do total) com apenas um indivíduo na amostragem; outras 56 (11,6 \%) ocorreram com apenas dois indivíduos, ou seja, 144 espécies $(29,5 \%$ do total $)$ podem ser consideradas como localmente raras.

\section{CONCLUSÃO}

Os testes estatísticos utilizados permitiram verificar a validação da amostra de dados para o emprego da técnica de análise multivariada e, portanto, para a construção do índice fitossociológico e socioeconômico - IFSE.

$\mathrm{O}$ índice proposto pode contribuir para as propostas que visam à recuperação de áreas degradadas pelas atividades agropecuárias e minerárias e, também, para a restauração de áreas de preservação permanente (APP) de reserva legal (RL), com base na indicação de espécies-chave, assim como da quantidade de mudas das mesmas, visando restaurar a paisagem primitiva.

TABELA 3: Relação das espécies de prioridade alta (em negrito) e média baseada no índice fitossociológico e socioeconômico, com respectivas abundâncias absolutas e relativas e número de mudas a plantar por hectare calculado e efetivo.

TABLE 3: List of high priority species (in bold) and average index calculated by phytosociological and socioeconomic status, with their absolute and relative abundances and number of seedlings planted per hectare, calculated and effective.

\begin{tabular}{|c|c|c|c|c|c|c|}
\hline \multirow{2}{*}{ Nome científico } & \multirow{2}{*}{ Nome popular } & \multirow{2}{*}{ CAT } & \multicolumn{2}{|c|}{ Abundância } & \multicolumn{2}{|c|}{$\mathrm{N}^{\mathrm{o}}$ mudas $\mathrm{ha}^{-1}$} \\
\hline & & & $\mathrm{N}^{\mathrm{o}}$ ind. $20 \mathrm{ha}^{-1}$ & $\%$ & Calculado & Efetivo \\
\hline Oenocarpus bacaba & bacaba & 1 & 1309 & 12,95 & 237,4 & 237 \\
\hline Geissopermum sericeum & quinarana & 1 & 377 & 3,73 & 68,4 & 68 \\
\hline Bertholletia excelsa & castanha-do-pará & 1 & 27 & 0,27 & 4,9 & 10 \\
\hline Pouteria oppositifolia & guajará-bolacha & 2 & 61 & 0,60 & 11,1 & 11 \\
\hline Tetragastris panamensis & barrotinho & 2 & 255 & 2,52 & 46,2 & 46 \\
\hline Aniba canelila & casca-preciosa & 2 & 39 & 0,39 & 7,1 & 10 \\
\hline Goupia glabra & cupiúba & 2 & 50 & 0,49 & 9,1 & 10 \\
\hline Bellucia grossularioides & muúba & 2 & 120 & 1,19 & 21,8 & 22 \\
\hline Hevea guianensis & seringa-itaúba & 2 & 116 & 1,15 & 21,0 & 21 \\
\hline Licaria rigida & louro-ferro & 2 & 125 & 1,24 & 22,7 & 23 \\
\hline Dipteryx odorata & cumarú & 2 & 14 & 0,14 & 2,5 & 10 \\
\hline Aspidosperma nitidum & carapanaúba-miúda & 2 & 36 & 0,36 & 6,5 & 10 \\
\hline Brosimum guianense & janitá & 2 & 87 & 0,86 & 15,8 & 16 \\
\hline Virola michelli & ucuúba-preta & 2 & 88 & 0,87 & 16,0 & 16 \\
\hline Swartzia recurva & gombeira-pé-de-anta & 2 & 60 & 0,59 & 10,9 & 11 \\
\hline Endopleura uchi & uchi & 2 & 59 & 0,58 & 10,7 & 11 \\
\hline Brosimum rubescens & muirapiranga & 2 & 53 & 0,52 & 9,6 & 10 \\
\hline Chimarrhis turbinata & pau-de-remo & 2 & 35 & 0,35 & 6,3 & 10 \\
\hline Protium crenata & breu-grande & 2 & 45 & 0,45 & 8,2 & 10 \\
\hline Protium tenuifolium & breu-preto & 2 & 105 & 1,04 & 19,0 & 19 \\
\hline Protium hebetatum & breu-mescla-vermelho & 2 & 110 & 1,09 & 19,9 & 20 \\
\hline Rinorea riana & jacamim-branco & 2 & 491 & 4,86 & 89,0 & 89 \\
\hline Eschweilera coriacea & matamatá-branco & 2 & 83 & 0,82 & 15,1 & 15 \\
\hline Pouteria gongrypii & abiu-vermelho folha-grande & 2 & 124 & 1,23 & 22,5 & 22 \\
\hline Lecythis prancei & jarana-amarela & 2 & 57 & 0,56 & 10,3 & 10 \\
\hline Totais & & - & 3.926 & 38,35 & 712,00 & 736 \\
\hline
\end{tabular}


A indicação do índice proposto neste trabalho como metodologia alternativa, em função do rigor científico e dos significantes resultados, deve ter aplicações em outras comunidades vegetais para efeito de validação do método.

\section{AGRADECIMENTOS}

À Mineração Rio do Norte-MRN, financiadora deste estudo, e a seus funcionários, em especial ao Júlio Sanna; ao José Risonei A. Silva e demais servidores da FLONA Saracá-Taquera/ ICMBio, ao Nélson A. Rosa e Vitor H. F. Gomes, expressamos nossos sinceros agradecimentos.

\section{REFERÊNCIAS BIBLIOGRÁFICAS}

BACHA, C. J. C.; BARROS, A. L. M. Reflorestamento no Brasil: evolução recente e perspectivas para o futuro. Scientia Florestalis, Piracicaba, v. 66, p. 191-203. 2004.

BRIENZA JÚNIOR, S. et al. Considerações sobre a recuperação de áreas alteradas por atividades agropecuária e florestal na Amazônia brasileira., Belém, 1995. p. 3-27p. (Documentos Embrapa/ Cpatu, v. 83)

BROWN, S. et al. Biomass estimation methods for tropical forests with applications to forest inventory data. Forest Science v. 35, n. 4, p. 881-902, 1989. CAMPOS, J. C. C.; LEITE, H. G. Mensuração florestal: perguntas e respostas. Viçosa: Universidade Federal de Viçosa, 2002. 407 p.

FARIA, J. M. R.; DAVIDE, A. C.; BOTELHO, S. A. Comportamento de espécies florestais em área degradada, com duas adubações de plantio. Cerne, Lavras, v. 3, n. 1, p. 25-77. 1997.

FRANCO, A. A. et al. Revegetação de solos degradados. Seropédica, 1992. 11, 3-11. (Comunicado Técnico Embrapa/Cnpab, v. 9).

GAMA-RODRIGUES, A. C. et al. Balanço de carbono e nutrientes em plantios puro e misto de espécies florestais nativas no sudeste da Bahia. Revista Brasileira de Ciência do Solo, Viçosa, v. 32, n. 3, p. 1165-1179, 2008.

GAUCH, H. G. Multivariate Analysis in Community Ecology. Cambridge: Cambridge Univ. Press, 1982. 298 p.

GOODALL, D. W. Objective methods for the classification of vegetation. III. An essay in the use of factor analysis. Austral J. Bot., v. 2, p. 304-324. 1954.

GUIMARÃES NETO, A. B. et al. Avaliação do plantio homogêneo de mogno, Swietenia macrophylla King, em comparação com o plantio consorciadoo com Eucalyptus urophylla S.T. Blake, após 40 meses de idade. Revista Árvore, Viçosa, v. 28, n. 6, p. 777-784, 2004.

HAIR JR, J. F. et al. Análise multivariada de dados. 5. ed. Porto Alegre: Bookman, 2005.

JOHNSON, R. A.. WICHERN, D. W. Applied Multivariate Statistical Analysis, 6th ed. New Jersey: Pearson, 2007. 761 p.

KAPPELLE, M. K. et al. Changes in diversity along a successional gradient in a Costa Rican upper montane. Biodiversity and Conservation, Amsterdan, v. 4, p. 10-34, 1995.

LA ROI, G. H. Classification and ordination of southern boreal forest form the Hondo - Slave Lake area of central Alberta. Canad. J. Bot., Montreal, v. 70, p. 614-628, 1992.

LAMB, D. et al. Restoration of degraded tropical forest landscapes. Science, New York, v. 310, p.1628-1632, 2005.

LAMB, D. Reforestation of degraded tropical forest lands in the Asia-Pasific region. Journal of Tropical Forest Science, Kepong, v. 7, n. 1, p. 1-7, 1994.

MCKAY, J. K. et al. "How Local Is Local?" A Review of Practical and Conceptual Issues in the Genetics of Restoration. Restoration Ecology, Washington, v. 13, n. 3, p. 432-440, 2005.

MINGOTI, S. A. Análise de dados através de métodos de estatística multivariada: uma abordagem aplicada. Belo Horizonte: Ed. UFMG, 2005. $297 \mathrm{p}$.

MOLINARO, L. C. Função ecológica de espécies arbóreas (Vismia guianensis, Inga edulis, Inga sp) na sucessão vegetal em áreas degradadas pela exploração petrolífera, na região de Urucu, AM. 2005. 83 f. Dissertação (Mestrado em Biologia Vegetal) - Instituto Nacional de Pesquisas da Amazônia, Manaus, 2005.

MOREIRA, P. R. Manejo do solo e recomposição da vegetação com vistas a recuperação de áreas degradadas pela extração de bauxita, Poços de Caldas, MG. 2004. 155 f. Tese (Doutorado em Ciências Biológicas) - Universidade Estadual Paulista, Rio Claro, 2004.

NAPPO, M. E. Dinâmica da regeneração natural de espécies arbóreas e arbustivas no sub-bosque de povoamentos de Mimosa scabrella Bentham, em área minerada, em Poços de Caldas-MG. 2002. 86 f. Tese (Doutorado em Ciências Florestais) - Universidade Federal de Viçosa, Viçosa, 2002.

OGUTU, Z. A. Multivariate analysis of plant 
communities in Narok district, Kenya: the influence of environmental factors and human disturbance. Vegetatio, v. 126, p. 181-189. 1996.

OLIVEIRA-FILHO, A. T. Comparison of the Woody flora and soils of six areas of montane semiciduous Forest in southern Minas Gerais, Brazil. J. Bot., Edinburgh, v. 51, n. 3, p. 355-389, 1994.

OLIVEIRA-FILHO, A. T.; RATTER, J. A. A study of the origin of central Brazilian forests by the analysis of plant species distribution patterns. J. Bot. Edinburgh, v. 52, n. 2, p.141-194. 1995. PARROTTA, J.; KNOWLES, O.H. Restoring tropical forests on land mined for bauxite: Examples from the Brazilian Amazon. Ecological Engineering, Columbus, v. 17, p. 219-239, 2001. PIELOU, E. C. The interpretation of ecological data: a primer on classification and ordination. New York: John Wiley \& Sons Publ., 1984. 263 p. RADAMBRASIL. Folha SA 22 - Belém. Rio Janeiro, Ministério das Minas e Energia, Departamento Nacional da Produção Mineral, Projeto Radambrasil, v. 5, 558 p. 1974.

RODRIGUES, R. R.; GANDOLFI, S. Conceitos, tendências e ações para a recuperação de florestas ciliares. In: RODRIGUES, R. R.; LEITÃO FILHO, H. R. (eds.) Matas ciliares: conservação e recuperação. 2. ed. São Paulo, Edusp, 2001, p. 235247.

SALOMÃO, R. P. et al. As florestas de Belo Monte na grande curva do rio Xingu, Amazônia Oriental. Belém, Bol. Mus. Para. Emílio Goeldi, Ciências Naturais, Belém, v. 2, n. 3, p. 57-153, set./dez. 2007.

SALOMÃO, R. P. etal.Dinâmica de reflorestamentos visando a restauração da paisagem florestal em áreas de mineração na Amazônia. Bol. Mus. Para. Emílio Goeldi, sér. Bot., Belém, v. 18, n. 1, p. 157194. 2002.

SALOMÃO, R. P. et al. Estrutura diamétrica e breve caracterização ecológica-econômica de 108 espécies arbóreas da floresta amazônica brasileira. Interciência, Caracas, v. 20, n. 1, p. 20-9. 1995. SALOMÃO, R. P., ROSA, N. A.; MORAIS, K. A. C.. Dinâmica da regeneração natural de árvores em áreas mineradas da Amazônia. Bol. Mus. Para. Emílio Goeldi, Ciências Naturais, Belém, v. 2, p.
85-139, 2007.

SANTANA, A. C. de. Índice de desempenho competitivo das empresas de polpa de frutas do Estado do Pará. Revista de Economia e Sociologia Rural, Brasília, v. 45, p. 749 - 775, 2007.

SANTOS, R. M. et al. Riqueza e similaridade florística de oito remanescentes florestais no norte de Minas Gerais, Brasil. Revista Árvore, Viçosa, v. 31, n. 1, p. 135-144, 2007.

SER. Society for Ecologial Restoration, Princípios da SER International sobre a restauração ecológica, 2004. SER and Tucson: Society for Ecological Restoration International. Disponível em: <(http://www.ser.org) $>$ Acesso em: 4 de outubro de 2010.

SHANLEY, P.; MEDINA G. Frutíferas e Plantas Úteis na Vida Amazônica. Belém, CIFOR:Imazon, 2005. 300 p.

SHANLEY, P.; ROSA, N. A. Conhecimento em erosão: um inventário etnobotânico na fronteira de exploração da Amazônia oriental. Bol. Mus. Para. Emílio Goeldi, Ciências Naturais, Belém, v. 1, n. 1, p. 147-71. 2005.

SILVA, A. F.; SHEPHERD, G. J. Comparações florísticas entre algumas matas em floresta densa da bacia do Rio Juruá. Bol. Mus. Paraense Emilio Goeld, sér.Bot., v. 8, n. 2, p. 203-258, 1986.

STELLA, A. Seleção de especies arboreas tropicais prioritarias para a conservação : 0 caso da Estação Ecologica de Caetetus. 2002. 73 f. Dissertação (Mestrado em Biologia Vegetal) Universidade de Campinas, Campinas. 2002.

TAGGART, J.B. Ordination as an aid in determiningpriorities forplantcommunityprotection. Biol. Conservation, San Diego, v. 68, p. 135-141. 1994.

TOLEDO, L. O. et al. Análise multivariada de atributos pedológicos e fitossociológicos aplicada na caracterização de ambientes de cerrado no norte de Minas Gerais. Revista Árvore, Viçosa, v. 33, n. 5, p. 957-968, 2009.

VIEIRA, I. C. G. et al. Bases técnicas e referenciais para o Programa de Restauração Florestal do Pará: Um Bilhão de Árvores para a Amazônia. Pará Desenvolvimento, Belém, v. 2, p. 9-103, 2009. 